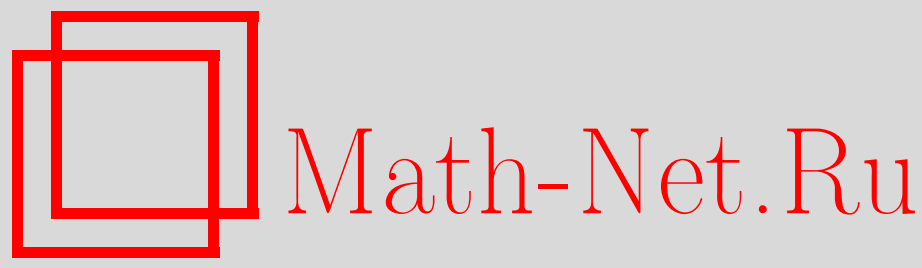

В. В. Жаринов, Формула Грина для разностных операторов, ТМФ, 2009, том 161, номер 2, 147-153

DOI: https://doi.org/10.4213/tmf6427

Использование Общероссийского математического портала Math-Net.Ru подразумевает, что вы прочитали и согласны с пользовательским соглашением http://www . mathnet.ru/rus/agreement

Параметры загрузки:

IP: 18.208 .226 .222

26 апреля 2023 г., 18:00:57

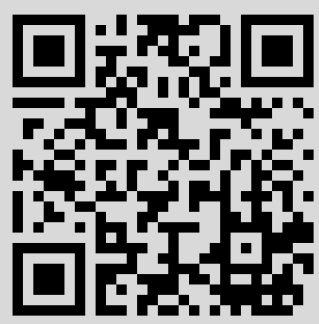




\section{ФОРМУЛА ГРИНА ДЛЯ РАЗНОСТНЫХ ОПЕРАТОРОВ}

Для разностных операторов на многомерной целочисленной решетке построен аналог классической формулы Грина для линейных операторов в частных производных.

Ключевые слова: формула Грина, целочисленная решетка, группа сдвигов, разностный оператор, разностный джет.

Формула Грина для уравнения Лапласа

$$
\phi \cdot \Delta(\psi)-\Delta(\phi) \cdot \psi=\sum_{\alpha} \partial_{\alpha}\left(J^{\alpha}(\phi, \psi)\right)
$$

для всех $\phi, \psi \in \mathcal{C}^{\infty}\left(\mathbb{R}^{D}\right)$, где $J^{\alpha}(\phi, \psi)=\phi \cdot \partial_{\alpha}(\psi)-\partial_{\alpha}(\phi) \cdot \psi$, и ее обобщения на другие уравнения классической математической физики широко используются в теории уравнений в частных производных. Фактически подобная формула Грина справедлива для любой линейной системы уравнений в частных производных с переменными коэффициентами (см., например, работу [1]). В рамках алгебро-геометрического подхода (группового анализа) она имеет естественное обобщение и на нелинейные системы уравнений в частных производных (см., например, работы [2], [3]). Ниже аналогичная формула доказана для разностных систем уравнений на многомерной целочисленной решетке.

Введем следующие обозначения:

1) $\mathbb{F}=\mathbb{R}, \mathbb{C}$ - числовое поле;

2) $\mathbb{Z}=\{0, \pm 1, \pm 2, \ldots\}, \mathbb{N}=\{1,2,3, \ldots\}$;

3) $\mathcal{A}$ - унитальная ассоциативная коммутативная $\mathbb{F}$-алгебра;

4) $\mathcal{M}, \mathcal{N}-\mathcal{A}$-модули.

\section{1. ЦЕЛОЧИСЛЕННАЯ РЕШЕТКА}

Для данного натурального $D \in \mathbb{N}$ положим $\mathbb{D}=\{1,2, \ldots, D\}$. Решетка $(D$-мерная целочисленная решетка) есть множество $\mathbb{L}=\mathbb{Z}^{D}$. На множестве $\mathbb{L}$ определены две алгебраические структуры: структура абелевой группы по сложению (группы сдви208)

$$
\mathbb{L} \ni m=\left(\mu^{1}, \ldots, \mu^{D}\right), n=\left(\nu^{1}, \ldots, \nu^{D}\right) \quad \mapsto \quad m+n=\left(\mu^{1}+\nu^{1}, \ldots, \mu^{D}+\nu^{D}\right) \in \mathbb{L}
$$

* Математический институт им. В. А. Стеклова РАН, Москва, Россия. E-mail: zharinov@mi.ras.ru 
и структура свободного $\mathbb{Z}$-модуля, где $\lambda m=\left(\lambda \mu^{1}, \ldots, \lambda \mu^{D}\right)$ для всех $\lambda \in \mathbb{Z}$ и всех $m=\left(\mu^{1}, \ldots, \mu^{D}\right) \in \mathbb{L}$, с базисом $e_{1}=(1,0, \ldots, 0), \ldots, e_{D}=(0, \ldots, 0,1)$, так что $m=\sum_{\alpha \in \mathbb{D}} \mu^{\alpha} e_{\alpha}$ для всех $m=\left(\mu^{1}, \ldots, \mu^{D}\right) \in \mathbb{L}$.

\section{2. ДЕЙСТВИЯ ГРУППЫ СДВИГОВ}

Говорят, что определено действие группь $G$ на множестве $X$, если задан морфизм групп $T: G \rightarrow \operatorname{Aut}(X)$, где $\operatorname{Aut}(X)$ - группа всех автоморфизмов множества $X$.

По построению определено действие $T: \mathbb{L} \rightarrow \operatorname{Aut}(\mathbb{L})$ группы сдвигов $\mathbb{L}$ на решеткe $\mathbb{L}$,

$$
\mathbb{L} \ni n \mapsto T_{n} \in \operatorname{Aut}(\mathbb{L}), \quad m \mapsto T_{n}(m)=m+n .
$$

На поле $\mathbb{F}$ группа сдвигов $\mathbb{L}$ действует тривиально: $T: \mathbb{L} \rightarrow \operatorname{Aut}(\mathbb{F})$, где $T_{n}(a)=a$ для всех $n \in \mathbb{L}, a \in \mathbb{F}$.

Говорят, что действие группъ сдвигов $\mathbb{L}$ определено на алгебре $\mathcal{A}$, если задан морфизм групп $T: \mathbb{L} \rightarrow \operatorname{Aut}_{\mathbb{F}}(\mathcal{A})$, где $\operatorname{Aut}_{\mathbb{F}}(\mathcal{A})$ - группа всех автоморфизмов алгебры $\mathcal{A}$, так что

$$
T_{n}(a \phi+b \psi)=a T_{n}(\phi)+b T_{n}(\psi), \quad T_{n}(\phi \cdot \psi)=T_{n}(\phi) \cdot T_{n}(\psi)
$$

для всех $n \in \mathbb{L}, a, b \in \mathbb{F}, \phi, \psi \in \mathcal{A}$. Обратим внимание, что для всякого дифференцирования $\zeta$ алгебры $\mathcal{A}$ имеем

$$
\zeta(a \phi+b \psi)=a \zeta(\phi)+b \zeta(\psi), \quad \zeta(\phi \cdot \psi)=\zeta(\phi) \cdot \psi+\phi \cdot \zeta(\psi) .
$$

Ниже будем считать фиксированными унитальную ассоциативную коммутативную алгебру $\mathcal{A}$ и действие группы сдвигов $\mathbb{L}$ на ней.

Говорят, что действие группъ сдвигов $\mathbb{L}$ определено на $\mathcal{A}$-модуле $\mathcal{M}$, если задан морфизм групп $T: \mathbb{L} \rightarrow \operatorname{Aut}_{\mathcal{A}}(\mathcal{M})$, где $\operatorname{Aut}_{\mathcal{A}}(\mathcal{M})$ - группа всех автоморфизмов модуля $\mathcal{M}$, так что

$$
T_{n}(\phi \cdot f+\psi \cdot g)=T_{n}(\phi) \cdot T_{n}(f)+T_{n}(\psi) \cdot T_{n}(g)
$$

для всех $n \in \mathbb{L}, \phi, \psi \in \mathcal{A}, f, g \in \mathcal{M}$.

Пусть действие группы сдвигов $\mathbb{L}$ определено на $\mathcal{A}$-модулях $\mathcal{M}$ и $\mathcal{N}$, и пусть $\mathcal{P}=\operatorname{Hom}_{\mathcal{A}}(\mathcal{M}, \mathcal{N})$ есть $\mathcal{A}$-модуль всех морфизмов из модуля $\mathcal{M}$ в модуль $\mathcal{N}$. Тогда на модуле $\mathcal{P}$ определено действие $T: \mathbb{L} \rightarrow \operatorname{Aut}_{\mathcal{A}}(\mathcal{P})$ группы сдвигов $\mathbb{L}$ правилом

$$
T_{n}(p f)=T_{n}(p) T_{n}(f)
$$

для всех $n \in \mathbb{L}, p \in \mathcal{P}, f \in \mathcal{M}$, где $p f \in \mathcal{N}$ - образ элемента $f$ относительно морфизма $p, T_{n}(p f) \in \mathcal{N}$ - образ элемента $p f$ относительно сдвига $T_{n}, T_{n}(p) \in \mathcal{P}$ образ морфизма $p$ относительно сдвига $T_{n}, T_{n}(f) \in \mathcal{M}$ - образ элемента $f$ относительно сдвига $T_{n}, T_{n}(p) T_{n}(f)$ - образ элемента $T_{n}(f)$ относительно морфизма $T_{n}(p)$. Коротко, $T_{n}(p)=T_{n} \circ p \circ T_{-n}$ для всех $n \in \mathbb{L}$ и $p \in \mathcal{P}$.

В частности, если действие группы сдвигов $\mathbb{L}$ определено на $\mathcal{A}$-модуле $\mathcal{M}$, то оно определено и на дуальном $\mathcal{A}$-модуле $\mathcal{M}^{*}=\operatorname{Hom}_{\mathcal{A}}(\mathcal{M}, \mathcal{A})$ правилом

$$
T_{n}(\langle\omega, f\rangle)=\left\langle T_{n}(\omega), T_{n}(f)\right\rangle
$$


для всех $n \in \mathbb{L}, \omega \in \mathcal{M}^{*}, f \in \mathcal{M}$, где $\langle\omega, f\rangle \in \mathcal{A}$ - спаривание элементов $\omega$ и $f$, $T_{n}(\langle\omega, f\rangle) \in \mathcal{A}$ - образ элемента $\langle\omega, f\rangle$ относительно сдвига $T_{n}, T_{n}(\omega) \in \mathcal{M}^{*}$ - образ элемента $\omega$ относительно сдвига $T_{n}, T_{n}(f) \in \mathcal{M}$ - образ элемента $f$ относительно сдвига $T_{n}$. Коротко, $T_{n}(\omega)=T_{n} \circ \omega \circ T_{-n}$ для всех $n \in \mathbb{L}$ и $\omega \in \mathcal{M}^{*}$.

\section{3. РАЗНОСТНЫЕ ОПЕРАТОРЫ}

Пусть $\mathcal{M}, \mathcal{N}$ - фиксированные $\mathcal{A}$-модули, на которых определено действие группы сдвигов $\mathbb{L}$. Разностным оператором из модуля $\mathcal{M}$ в модуль $\mathcal{N}$ назовем всякое линейное отображение $P=\sum_{m \in \mathbb{L}} p^{m} \circ T_{m}$ из $\mathcal{M}$ в $\mathcal{N}$, где лишь конечное число коэффициентов $p^{m} \in \mathcal{P}=\operatorname{Hom}_{\mathcal{A}}(\mathcal{M}, \mathcal{N})$ ненулевые, так что $P(f)=\sum_{m} p^{m} T_{m}(f)$ для всех $f \in \mathcal{M}$.

Каждому разностному оператору $P=\sum_{m} p^{m} \circ T_{m}$ из модуля $\mathcal{M}$ в модуль $\mathcal{N}$ ставится в соответствие его формалъно сопряженный (по Лагранжу) разностный оператор $P^{*}=\sum_{m} T_{-m} \circ\left(p^{m}\right)^{*}$, действующий из дуального модуля $\mathcal{N}^{*}$ в дуальный модуль $\mathcal{M}^{*}$, где дуальные коэффициенты $\left(p^{m}\right)^{*} \in \mathcal{P}^{*}=\operatorname{Hom}_{\mathcal{A}}\left(\mathcal{N}^{*}, \mathcal{M}^{*}\right)$ определены правилом

$$
\left\langle p^{*} \omega, f\right\rangle=\langle\omega, p f\rangle
$$

для всех $p \in \mathcal{P}, \omega \in \mathcal{N}^{*}, f \in \mathcal{M}$. Для простоты введем удобное обозначение $\omega p=p^{*} \omega$ для всех $\omega \in \mathcal{N}^{*}$ и $p \in \mathcal{P}$. В частности, в таких обозначениях

$$
P^{*}(\omega)=\sum_{m} T_{-m}\left(\omega p^{m}\right)
$$

для всех $\omega \in \mathcal{N}^{*}$.

\section{4. ФОРМУЛА ГРИНА}

Разностные операторы с целочисленными коэффициентами определены на всех абелевых группах, на которых определены сдвиги. В частности, определены элементарные разностные операторы

$$
\Delta_{\alpha}=T_{e_{\alpha}}-1, \quad \alpha \in \mathbb{D}, \quad 1=\mathrm{id}=T_{0} .
$$

Обратим внимание, что

$$
\begin{aligned}
\Delta_{\alpha}(\phi \cdot \psi) & =\Delta_{\alpha}(\phi) \cdot \psi+\phi \cdot \Delta_{\alpha}(\psi)+\Delta_{\alpha}(\phi) \cdot \Delta_{\alpha}(\psi) \neq \\
& \neq \Delta_{\alpha}(\phi) \cdot \psi+\phi \cdot \Delta_{\alpha}(\psi), \quad \phi, \psi \in \mathcal{A},
\end{aligned}
$$

так что разностные операторы $\Delta_{\alpha}$ не являются дифференцированиями алгебры $\mathcal{A}$.

Лемма 1. Для любых $\mu \in \mathbb{Z} u \alpha \in \mathbb{D}$ имеет место равенство

$$
T_{\mu e_{\alpha}}-1=\Delta_{\alpha} \circ S_{\mu}^{\alpha}, \quad S_{\mu}^{\alpha}= \begin{cases}1+T_{e_{\alpha}}+\cdots+T_{(\mu-1) e_{\alpha}}, & \mu>0 \\ 0, & \mu=0 \\ -\left(T_{\mu e_{\alpha}}+\cdots+T_{-e_{\alpha}}\right), & \mu<0 .\end{cases}
$$

ДОКАЗАТЕЛЬСТво проводится прямыми вычислениями. 
Для каждого $m=\left(\mu^{1}, \ldots, \mu^{D}\right) \in \mathbb{L}$ положим

$$
\begin{array}{llll}
m_{0}=0, & m_{1}=\left(\mu^{1}, 0, \ldots, 0\right), & \ldots, & m_{D}=\left(\mu^{1}, \ldots, \mu^{D}\right)=m, \\
\bar{m}_{0}=m, & \bar{m}_{1}=\left(0, \mu^{2}, \ldots, \mu^{D}\right), & \ldots, & \bar{m}_{D}=0,
\end{array}
$$

так что $m_{\alpha}+\bar{m}_{\alpha}=m$ для всех $0 \leqslant \alpha \leqslant D$.

ЛЕмма 2. Для каждого $m \in \mathbb{L}$ существуют разностные операторы $J_{m}^{\alpha}, \alpha \in \mathbb{D}$, такие, что $T_{m}-1=\sum_{\alpha \in \mathbb{D}} \Delta_{\alpha} \circ J_{m}^{\alpha}$. Например, можно взять $J_{m}^{\alpha}=L_{m}^{\alpha}=T_{m_{\alpha-1}} \circ S_{\mu^{\alpha}}^{\alpha}$ или $J_{m}^{\alpha}=R_{m}^{\alpha}=T_{\bar{m}_{\alpha}} \circ S_{\mu^{\alpha}}^{\alpha}$.

ДокАЗАТЕльство. Действительно, в силу леммы 1

$$
\begin{aligned}
\sum_{\alpha} \Delta_{\alpha} \circ L_{m}^{\alpha} & =\sum_{\alpha}\left(T_{e_{\alpha}}-1\right) \circ T_{m_{\alpha-1}} \circ S_{\mu^{\alpha}}^{\alpha}=\sum_{\alpha} T_{m_{\alpha-1}} \circ\left(T_{e_{\alpha}}-1\right) \circ S_{\mu^{\alpha}}^{\alpha}= \\
& =\sum_{\alpha} T_{m_{\alpha-1}} \circ\left(T_{\mu^{\alpha} e_{\alpha}}-1\right)=\sum_{1 \leqslant \alpha \leqslant D}\left(T_{m_{\alpha}}-T_{m_{\alpha-1}}\right)=T_{m}-1 .
\end{aligned}
$$

Аналогичным образом проверяется утверждение леммы для $J_{m}^{\alpha}=R_{m}^{\alpha}$.

Теорема. Пусть $P=\sum_{m} p^{m} \circ T_{m}-$ разностный оператор, действующий из модуля $\mathcal{M}$ в модуль $\mathcal{N}$, и $P^{*}=\sum_{m} T_{-m} \circ\left(p^{m}\right)^{*}$ - его формально сопряженньй. Тогда существует билинейный разностный оператор $J=\left(J^{\alpha}\right): \mathcal{N}^{*} \times \mathcal{M} \rightarrow \mathcal{A}^{\mathbb{D}}$ такой, что для всех $\omega \in \mathcal{N}^{*}, f \in \mathcal{M}$, справедлива формула Грина

$$
\langle\omega, P(f)\rangle-\left\langle P^{*}(\omega), f\right\rangle=\sum_{\alpha} \Delta_{\alpha}\left(J^{\alpha}(\omega, f)\right) .
$$

Например, можно положить $J^{\alpha}(\omega, f)=\sum_{m} J_{m}^{\alpha}\left(\left\langle T_{-m}\left(\omega p^{m}\right), f\right\rangle\right)$, где $J_{m}^{\alpha}$ - разностные операторы, удовлетворяющие лемме 2.

ДокАЗАТЕльство. Действительно, в силу леммы 2

$$
\begin{aligned}
\sum_{\alpha} \Delta_{\alpha}\left(J^{\alpha}(\omega, f)\right) & =\sum_{\alpha} \sum_{m}\left(\Delta_{\alpha} \circ J_{m}^{\alpha}\right)\left(\left\langle T_{-m}\left(\omega p^{m}\right), f\right\rangle\right)= \\
& =\sum_{m} \sum_{\alpha}\left(\Delta_{\alpha} \circ J_{m}^{\alpha}\right)\left(\left\langle T_{-m}\left(\omega p^{m}\right), f\right\rangle\right)= \\
& =\sum_{m}\left(T_{m}-1\right)\left(\left\langle T_{-m}\left(\omega p^{m}\right), f\right\rangle\right)= \\
& =\sum_{m}\left(\left\langle\omega, p^{m} T_{m}(f)\right\rangle-\left\langle T_{-m}\left(\omega p^{m}\right), f\right\rangle\right)=\langle\omega, P(f)\rangle-\left\langle P^{*}(\omega), f\right\rangle .
\end{aligned}
$$

\section{5. ПРИМЕРЫ}

Пример 1. Разностный оператор Лапласа $\Lambda=\sum_{\alpha}\left(T_{e_{\alpha}}-2+T_{-e_{\alpha}}\right)$ действует на алгебре $\mathcal{A}$. Здесь $\Lambda^{*}=\Lambda$, и разностная формула Грина имеет вид

$$
\phi \cdot \Lambda(\psi)-\Lambda(\phi) \cdot \psi=\sum_{\alpha} \Delta_{\alpha}\left(J^{\alpha}(\phi, \psi)\right)
$$

для всех $\phi, \psi \in \mathcal{A}$, где $J^{\alpha}(\phi, \psi)=-\phi \cdot T_{-e_{\alpha}}(\psi)+T_{-e_{\alpha}}(\phi) \cdot \psi$. 
Пример 2. Множество $\mathcal{M}=\mathcal{A}^{M}, M \in \mathbb{N}$, обладает естественной структурой $\mathcal{A}$-модуля с покомпонентными операциями. Его элементы суть столбцы $f=\left(f^{\mu}\right)=$ $\left(f^{\mu} \in \mathcal{A} \mid 1 \leqslant \mu \leqslant M\right)$. Группа сдвигов $\mathbb{L}$ действует на $\mathcal{A}$-модуле $\mathcal{M}$ покомпонентно, $T_{n}(f)=\left(T_{n}\left(f^{\mu}\right)\right)$ для всех $n \in \mathbb{L}$. Дуальный $\mathcal{A}$-модуль $\mathcal{M}^{*}=\mathcal{A}_{M}$ состоит из строк $\chi=\left(\chi_{\mu}\right)$, спаривание $\langle\chi, f\rangle=\sum_{\mu} \chi_{\mu}, f^{\mu}=\chi f$ (умножение строки на столбец), группа сдвигов $\mathbb{L}$ действует на $\mathcal{M}^{*}$ также покомпонентно, $T_{n}(\chi)=\left(T_{n}\left(\chi_{\mu}\right)\right)$. Пусть $\mathcal{M}=\mathcal{A}^{M}, \mathcal{N}=\mathcal{A}^{N}$ есть пара $\mathcal{A}$-модулей указанного типа, тогда $\mathcal{A}$-модуль $\mathcal{P}=\operatorname{Hom}_{\mathcal{A}}(\mathcal{M}, \mathcal{N}) \simeq \mathcal{A}_{M}^{N}$ состоит из матриц $p=\left(p_{\mu}^{\nu}\right)$, причем образ $g=p f \in \mathcal{N}$ вычисляется по правилу $g^{\nu}=\sum_{\mu} p_{\mu}^{\nu} \cdot f^{\mu}$ (умножение матрицы на столбец). Группа сдвигов $\mathbb{L}$ действует на $\mathcal{A}$-модуле $\mathcal{P}$ покомпонентно, $T_{n}(p)=\left(T_{n}\left(p_{\mu}^{\nu}\right)\right)$ для всех $n \in \mathbb{L}$. Дуальный $\mathcal{A}$-модуль $\mathcal{P}^{*}=\operatorname{Hom}_{\mathcal{A}}\left(\mathcal{N}^{*}, \mathcal{M}^{*}\right) \simeq \mathcal{A}_{M}^{N}$ также состоит из матриц $p=\left(p_{\mu}^{\nu}\right)$, причем образ $\chi=\omega p$ вычисляется по правилу $\chi_{\mu}=\sum_{\nu} \omega_{\nu} \cdot p_{\mu}^{\nu}$ (умножение строки на матрицу). В таких обозначениях

$$
\langle\omega, p f\rangle=\sum_{\mu, \nu} \omega_{\nu} \cdot p_{\mu}^{\nu} \cdot f^{\mu}=\left\langle p^{*} \omega, f\right\rangle=\omega p f
$$

для всех $\omega \in \mathcal{N}^{*}, p \in \mathcal{P}, f \in \mathcal{M}$. Пусть $P=\sum_{m} p^{m} \circ T_{m}$ - разностный оператор из $\mathcal{A}$-модуля $\mathcal{M}=\mathcal{A}^{M}$ в $\mathcal{A}$-модуль $\mathcal{N}=\mathcal{A}^{N}$, и $P^{*}=\sum_{m} T_{-m} \circ\left(p^{m}\right)^{*}$ - его формально сопряженный. Тогда формула Грина для него имеет вид

$$
\omega P(f)-P^{*}(\omega) f=\sum_{\alpha} \Delta_{\alpha}\left(J^{\alpha}(\omega, f)\right)
$$

для всех $\omega \in \mathcal{N}^{*}, f \in \mathcal{M}$, где $J^{\alpha}(\omega, f)=\sum_{m} J_{m}^{\alpha}\left(T_{-m}\left(\omega p^{m}\right) f\right), J_{m}^{\alpha}$ - произвольные разностные операторы, удовлетворяющие лемме 2.

Пример 3. Пусть $\mathcal{A}(\mathbb{L})=\operatorname{Hom}(\mathbb{L}, \mathbb{F})$ - унитальная ассоциативная коммутативная алгебра всех $\mathbb{F}$-значных функций на решетке $\mathbb{L}$. Группа сдвигов $\mathbb{L}$ действует на алгебре $\mathcal{A}(\mathbb{L})$ стандартным образом:

$$
T_{n}(\phi)(m)=\phi\left(T_{n}(m)\right)=\phi(m+n)
$$

для всех $m, n \in \mathbb{L}, \phi \in \mathcal{A}(\mathbb{L})$. Такое определение и конструкция, приведенная в примере 2 , охватывают большую часть применений разностных операторов в численных экспериментах.

ПримеР 4. Пусть $V$ - конечномерное $\mathbb{F}$-линейное пространство. Определено тривиальное векторное расслоение

$$
\pi: \mathbb{L} \times V \rightarrow \mathbb{L}, \quad(m, u) \mapsto m
$$

с пространством расслоения $\mathbb{L} \times V$ и базой $\mathbb{L}$. Множество всех его сечений $\mathcal{F}(\pi)=$ $\operatorname{Hom}(\mathbb{L}, V)$ (т.е. отображений из $\mathbb{L}$ в $V$ ) есть $\mathcal{A}(\mathbb{L})$-модуль, на котором группа сдвигов действует стандартным образом:

$$
T_{n}(\phi)(m)=\phi\left(T_{n}(m)\right)=\phi(m+n)
$$

для всех $m, n \in \mathbb{L}, \phi \in \mathcal{F}(\pi)$. 
Расслоение разностных джетов (подробнее см. работу [4]) есть тривиальное векторное расслоение

$$
\pi^{\infty}: \mathbb{L} \times \mathbf{V} \rightarrow \mathbb{L}, \quad(m, \mathbf{u}) \mapsto m
$$

с базой $\mathbb{L}$ и пространством расслоения (пространством разностных джетов) $\mathbb{L} \times \mathbf{V}$, где типичный слой

$$
\mathbf{V}=V_{\mathbb{L}}=\left\{\mathbf{u}=\left(u_{k}\right) \mid u_{k} \in V, k \in \mathbb{L}\right\} .
$$

Группа сдвигов $\mathbb{L}$ действует на типичном слое $\mathbf{V}$ по естественному правилу $T_{n}(\mathbf{u})=\left(u_{T_{n}(k)}\right)=\left(u_{k+n}\right)$, а на пространстве разностных джетов $\mathbb{L} \times \mathbf{V}$ - по правилу $T_{n}((m, \mathbf{u}))=\left(T_{n}(m), T_{n}(\mathbf{u})\right)$ для всех $k, m, n \in \mathbb{L}, \mathbf{u}=\left(u_{k}\right) \in \mathbf{V}$. Множество $\mathcal{F}\left(\pi^{\infty}\right)=\operatorname{Hom}(\mathbb{L}, \mathbf{V})$ всех сечений расслоения $\pi^{\infty}$ (т.е. отображений из $\mathbb{L}$ в $\mathbf{V}$ ) есть $\mathcal{A}(\mathbb{L})$-модуль, на котором группа сдвигов $\mathbb{L}$ действует по индуцированному правилу $T_{n} \circ T_{n}(\boldsymbol{\varphi})=\boldsymbol{\varphi} \circ T_{n}$, так что $T_{n}(\boldsymbol{\varphi})=T_{-n} \circ \boldsymbol{\varphi} \circ T_{n}$, точнее

$$
\left(T_{n}(\varphi)\right)_{k}(m)=\phi_{k-n}(m+n)
$$

для всех $\boldsymbol{\varphi}=\left(\phi_{k} \in \mathcal{F}(\pi)\right), k, m, n \in \mathbb{L}$. Определено вложение $\mathbf{j}: \mathcal{F}(\pi) \rightarrow \mathcal{F}\left(\pi^{\infty}\right)$, $\phi \mapsto \mathbf{j}(\phi)$, где образ $\mathbf{j}(\phi)$ называется разностным джетом сечения $\phi$ и вычисляется по правилу $(\mathbf{j}(\phi))_{k}=T_{k}(\phi)$ для всех $k \in \mathbb{L}$. Эта конструкция характеризуется следующими свойствами:

1) сечение $\varphi \in \mathcal{F}\left(\pi^{\infty}\right)$ является джетом некоторого сечения $\phi \in \mathcal{F}(\pi), \varphi=$ $\mathbf{j}(\phi)$, тогда и только тогда, когда оно инвариантно относительно сдвигов, т.е. когда $T_{n} \varphi=\varphi$ для всех $n \in \mathbb{L}$;

2) для любой точки $(m, \mathbf{u}) \in \mathbb{L} \times \mathbf{V}$ существует единственное сечение $\phi \in \mathcal{F}(\pi)$ такое, что $\mathbf{j}(\phi)(m)=\mathbf{u}$.

Пусть $\mathcal{A}(\mathbb{L} \times \mathbf{V})=\operatorname{Hom}(\mathbb{L} \times \mathbf{V}, \mathbb{F})$ - унитальная ассоциативная коммутативная алгебра всех $\mathbb{F}$-значных функций на пространстве разностных джетов $\mathbb{L} \times \mathbf{V}$. Группа сдвигов $\mathbb{L}$ действует на алгебре $\mathcal{A}(\mathbb{L} \times \mathbf{V})$ по правилу $T_{n}(f)=f \circ T_{n}$, точнее

$$
T_{n}(f)(m, \mathbf{u})=f\left(T_{n}(m), T_{n}(\mathbf{u})\right)
$$

для всех $f \in \mathcal{A}(\mathbb{L} \times \mathbf{V}), m, n \in \mathbb{L}, \mathbf{u} \in \mathbf{V}$. Для каждого сечения $\varphi \in \mathcal{F}\left(\pi^{\infty}\right)$ определен морфизм алгебр (подстановка) $\mathcal{A}(\mathbb{L} \times \mathbf{V}) \rightarrow \mathcal{A}(\mathbb{L}),\left.f \mapsto f\right|_{\boldsymbol{\varphi}}$, где $\left(\left.f\right|_{\boldsymbol{\varphi}}\right)(m)=$ $f(m, \varphi(m))$ для всех $m \in \mathbb{L}$. Имеет место правило полного сдвига: для данного сечения $\varphi=\left(\phi_{k}\right) \in \mathcal{F}\left(\pi^{\infty}\right)$ равенство $\left.T_{n}(f)\right|_{\varphi}=T_{n}\left(\left.f\right|_{\varphi}\right)$ справедливо для всех $n \in \mathbb{L}$ u $f \in \mathcal{A}(\mathbb{L} \times \mathbf{V})$ тогда и только тогда, когда $\boldsymbol{\varphi}=\mathbf{j}(\phi)$, где $\phi=\phi_{0}$.

В приложениях (групповой анализ разностных и дифференциально-разностных уравнений, разностное вариационное исчисление, см., например, работы [4]-[7]) обычно используется подалгебра алгебры $\mathcal{A}(\mathbb{L} \times \mathbf{V})$, состоящая из всех функций $f=f(m, \mathbf{u}) \in \mathcal{A}(\mathbb{L} \times \mathbf{V})$, при каждом фиксированном $m \in \mathbb{L}$ гладко зависящих $\left(\right.$ т.е. класса $\mathcal{C}^{\infty}$ ) лишь от конечного числа разностных переменных $\mathbf{u}=\left(u_{k}\right)$ (подробнее см., например, работу [4]). Эта подалгебра и конструкция из примера 2 полностью обеспечивают упомянутые выше приложения.

Благодарности. Работа выполнена при финансовой поддержке РФФИ (грант № 07-01-00144_а) и Программы поддержки ведущих научных школ (грант НШ$3224.2008 .1)$. 


\section{Список литературы}

[1] В. С. Владимиров, В. В. Жаринов, Дифферени. уравнения, 16:5 (1980), 845-867.

[2] В. В. Жаринов, ДАН СССР, 296:4 (1987), 785-787.

[3] V. V. Zharinov, Lecture Notes on Geometrical Aspects of Partial Differential Equations, Ser. Sov. and East Eur. Math., 9, World Sci., Singapore, 1992.

[4] В. В. Жаринов, ТМФ, 157:3 (2008), 391-405.

[5] В. Э. Адлер, А. Б. Шабат, Р. И. Ямилов, ТМФ, 125:3 (2000), 355-424.

[6] P. E. Hydon, E. L. Mansfield, Found. Comput. Math., 4:2 (2004), 187-217.

[7] W. Hereman, M. Colagrosso, R. Sayers, A. Ringler, B. Deconinck, M. Nivala, M. Hickman, "Continuous and discrete homotopy operators and the computation of conservation laws", Differential Equations with Symbolic Computation, Trends Math., Birkhäuser, Basel, 2005, 255-290.

Поступила в редакцию 7.05.2009 